\title{
List of type-specimens of Isoptera (Insecta) in the collection of the Museu Paraense Emílio Goeldi, State of Pará, Brazil
}

\author{
Agno Nonato Serrão ACIOLI ${ }^{1}$, Maria Lucia Jardim MACAMBIRA
}

\section{ABSTRACT}

The collection of the Museu Paraense Emílio Goeldi (MPEG), in the city of Belém, State of Pará, Brazil, has 65 samples of type-specimens of Isoptera, representing 26 species ( 21 holotypes and 5 paratypes) of 18 genera. This paper lists the number of specimens of each caste in each type series, type localities with geographical coordinates, collectors, and dates of collection.

\section{KEY-WORDS}

Termites, Amazonia, Collection, Isoptera, Types

\section{Lista dos exemplares-tipos de Isoptera (Insecta) na coleção do Museu Paraense Emílio Goeldi, Estado do Pará, Brasil}

\section{RESUMO}

A Coleção do Museu Paraense Emílio Goeldi (MPEG), na cidade de Belém, Estado do Pará, Brasil, possui 65 amostras que são exemplares-tipos de Isoptera, representando 26 espécies (21 holótipos e 5 parátipos) pertencentes a 18 gêneros. Este trabalho lista o número de espécimes de cada casta em cada série-tipo, localidade tipo com as coordenadas geográficas, coletor e dados da coleta.

PALAVRAS-CHAVE

Cupins, Amazônia, Coleções, Isoptera, Tipos

UFAM/ Instituto Natureza e Cultura-Inc, Curso de Ciências Agrárias e Ambientais, Bejamim Constant-AM. Rua Primeiro de Maio s/n. Colônia. CEP: 69630-000 - Benjamin Constant, AM - Brasil. Telefone: (97) 3415 5677. e-mail: acioli@ufam.edu.br 
The Museu Paraense Emílio Goeldi was founded in 1866. It is one the oldest scientific museums in Brazil and has the largest collection of specimens from the Brazilian Amazon Region. The invertebrate collection of the MPEG contains approximately two million specimens, of which about 2200 are type-specimens (holotypes and paratypes) of more than 300 species of insects. The Isoptera collection has 4191 lots in alcohol, with 179 identified species of 60 genera and 4 families. The list of the holotypes and paratypes of termites in the MPEG collection, presented here, follows the recommendation of the International Code of Zoological Nomenclature (ICZN, see Ride et al. 1999), which calls for the publication of type catalogs and lists.

In the list, the names of families, subfamilies, genera and species are presented in alphabetical order. The information listed for each species includes:

Name of the species: author or authors, year of publication (as in the bibliography).

Type material (holotype or paratype): caste that represents the holotype, number of the type-colony in the collection of the MPEG, type-locality (Country, State, City, other information as river, farm, etc.), collector or collectors, date of collection, castes and number of specimens of each caste (in case of alates or dealates the number of males and females is also given). Label information given verbatim is presented within quotation marks.

The sign (=) indicates a synonym, the currently valid name being that after the sign. Name between [ ] means the old denomination of the locality.

Abbreviations used: AM, AP, MT, and PA, correspond to the Brazilian States of Amazonas, Amapá, Mato Grosso and Pará, respectively; "APEG”, “Área de Pesquisa Ecológica do rio Guamá” (Guamá River Ecological Research Area), a forest reserve now within the "Área de Proteção Ambiental de Belém" (Belém Environmental Protection Area) in the Municipality of Belém, near the Guamá river; "sd", "sem data" (without date); "det.”, "determined”; "E. E.”, "ecologyc station”. Geographical coordinates of type-localities were obtained directly from the labels, from the published work where the taxon is described or from official maps and their gazetteers.

The MPEG invertebrate collection contains 65 samples of type-specimens of Isoptera, of which 21 are holotypes and 5 paratypes. In total, 26 species are represented by types, grouped in 18 genera, three subfamilies and two families of termites. The collection of types is separated from the general collection of termites. The samples, each in a small glass container filled with $70 \%$ ethanol, are stored in larger glasses with plastic covers also filled with ethanol. In Table 1, type-localities and their respective geographical coordinates are given.
FAMILY RHINOTERMITIDAE

Subfamily Rhinotermitinae

\section{Genus Dolichorhinotermes Emerson}

- japuraensis Constantino, 1990a

Holotype: Major soldier. MPEG 2853. "BRASIL, AM, Maraã, rio Japurá, R. Constantino, 13.X.1988”.

Paratypes: MPEG 2853. The same data as the holotype: 4 major soldiers, 37 smaller soldiers, 54 workers.

\section{FAMILY TERMITIDAE}

Subfamily Nasutitermitinae

Genus Agnathotermes Snyder

- crassinasus Constantino, 1990b

Holotype: Soldier. MPEG 2865. "BRASIL, AM, Maraã, rio Japurá, R. Constantino, 13.X.1988”.

Paratypes: MPEG 2865. The same data as the holotype: 2 soldiers, 7 workers.

\section{Genus Anhangatermes Constantino}

- macarthuri Constantino, 1990c

Holotype: Soldier. MPEG 3267. "BRASIL, AP, Serra do Navio, R. Constantino, 02.XI.1989”.

Paratypes: MPEG 3267. The same data as the holotype: 13 soldiers, 52 workers.

\section{Genus Araujotermes Fontes}

- nanus Constantino, 1991a

Holotype: Soldier. MPEG 2945. "BRASIL, AM, Maraã, rio Japurá, R. Constantino, 27.X.1988”.

Paratypes: MPEG 2945. The same data as the holotype: 35 soldiers, 32 workers.

\section{Genus Armitermes Wasmann}

- gnomus Constantino, 1991a

Holotype: Soldier. MPEG 2903. "BRASIL, AM, Maraã, rio Japurá, R. Constantino, 21.X.1988”.

Paratypes: MPEG 2903. The same data as the holotype: 13 soldiers, 64 workers, 34 imagoes (22 §̃, 12 क); MPEG 2858. "BRASIL, AM, Maraã, rio Japurá, R. Constantino, 13.X.1988”: 23 soldiers, 58 workers; MPEG 2854. Idem: 33 soldiers, 48 workers; MPEG 2841. "BRASIL, AM, Maraã, rio Japurá, R. Constantino, 12.X.1988”: 1 soldier.

\section{Genus Cyranotermes Araujo}

- caete Cancello, 1987

Paratypes: MPEG 3310. "BRASIL, PA, Serra Norte, Carajás, R. Constantino,VII-VIII.1985”: 2 soldiers, 3 workers.

- glaber Constantino, 1990d

Holotype: Soldier. MPEG 3250. "BRASIL, AP, Macapá, Parque Zoobotânico, R. Constantino, 29.X.1989”. 
Paratypes: MPEG 3250. The same data as the holotype: 14 soldiers, 34 workers; MPEG 3237. "BRASIL, AP, Macapá, Parque Zoobotânico, R. Constantino, 28.X.1989”: 22 soldiers, 50 workers.

Genus Embiratermes Fontes

- ignotus Constantino, 1991a

Holotype: Soldier. MPEG 2879. "BRASIL, AM, Maraã, rio Japurá, R. Constantino, 15.X.1988”.

Paratypes: MPEG 2879. The same data as the holotype: 13 workers.

- parvirostris Constantino, 1992a

Holotype: Soldier. MPEG 3203. "BRASIL, AP, Macapá, Parque Zoobotânico, R. Constantino, 22.X.1989”.

Paratypes: MPEG 3203. The same data as the holotype: 15 soldiers, 50 workers; MPEG 3151. "BRASIL, AP, Macapá, Parque Zoobotânico, R. Constantino, 17.X.1989”: 11 soldiers, 41 workers.

- robustus Constantino, 1992a

Holotype: Soldier. MPEG 3276. "BRASIL, AP, Serra do Navio, R. Constantino, 02.XI.1989”.

Paratypes: MPEG 3276. The same data as the holotype: 10 soldiers, 58 workers.

\section{Genus Ereymatermes Constantino}

- rotundiceps Constantino, 1991b

Holotype: Soldier. MPEG 2790. "BRASIL, AM, Maraã, rio Japurá, R. Constantino, 06.X.1988”.

Paratypes: MPEG 2790. The same data as the holotype: 13 soldiers, 55 workers, 19 imagoes (12 ðં, 7 9); MPEG 2788. The same data as the holotype: 39 soldiers, 41 workers, 2 imagoes ( 1 ô, 1 ㅇ).

\section{Genus Ibitermes Fontes}

- tellustris Constantino, 1990a

Holotype: Soldier. MPEG 2833. "BRASIL, AM, Maraã, rio Japurá, R. Constantino, 12.X.1988”.

Paratypes: MPEG 2833. The same data as the holotype: 8 soldiers, 20 workers; MPEG 2888. "BRASIL, AM, Maraã, rio Japurá, R. Constantino, 18.X.1988”: 13 soldiers, 14 workers; MPEG 2829. “BRASIL, AM, Maraã, 1051'S/65027'W, R. Constantino, 11.X.1988": 1 soldier, 19 workers.

Genus Labiotermes Holmgren

- guasu Constantino \& Acioli, 2006 (In Constantino et al., 2006)

Paratypes: MPEG 1331. "BRASIL, MT, E. E. Ique-Juruema, A.E. Mill, 22.IX.1980": 5 Soldiers, 17 workers.

Genus Nasutitermes Dudley

- acangussu Bandeira \& Fontes, 1979
Holotype: Soldier. MPEG 331. "BRASIL, AM, Itacoatiara [Silves], fazenda Aruanã, Km-232, rodovia Manaus - Itacoatiara, $03^{0} 04^{\prime}$ S, 58 $45^{\prime} \mathrm{W}, \mathrm{A} . \mathrm{G}$. Bandeira, 11.IV.1978”.

Paratypes: MPEG 540. "BRASIL, PA, Itaituba, Parque Nacional da Amazônia, S.A.Marques, 23.I.1979”: 55 soldiers, 40 workers; MPEG 337. "BRASIL, AM, rodovia Manaus-Itacoatiara, Km-232, capoeira, A.G. Bandeira, 12.IV.1978": 19 soldiers, 6 workers; MPEG 333. "BRASIL, AM, rodovia Manaus-Itacoatiara, Km-232, A.G.Bandeira, 11.VI.1978": 13 soldiers, 20 workers; MPEG 331. The same data as the holotype: 60 soldiers, 7 workers; MPEG 256. "BRASIL, AM, Itacoatiara [Silves], AM-010, Km232, pastagem, A.G.Bandeira, 04.IX.1977”: 20 soldiers, 21 workers; MPEG 242. "BRASIL, AM, Itacoatiara [Silves], AM-010, Km-232, pastagem, A.G. Bandeira, 14.X.1977”: 9 Soldiers, 11 workers; MPEG 229. "BRASIL, AM, Itacoatiara [Silves], AM-010, Km-232, pastagem, A.G.Bandeira, 29.IX.1977": 15 soldiers, 7 workers, 6 imaturos; MPEG 198. "BRASIL, AM, rodovia Manaus-Itacoatiara, Km-232, capoeira, A.G.Bandeira, 10.VI.1977”: 24 soldiers; MPEG 192. "BRASIL, AM, rodovia Manaus-Itacoatiara, Km-232, capoeira, A.G.Bandeira, 10.VI.1977”: 22 soldiers; MPEG 191. "BRASIL, AM, rodovia Manaus-Itacoatiara, Km-232, capoeira, A.G.Bandeira, 10.VI.1977”: 31 soldiers; MPEG 189. "BRASIL, AM, rodovia Manaus-Itacoatiara, Km-232, capoeira, A.G.Bandeira, 10.VI.1977”: 38 soldiers, 1 worker; MPEG 171. "BRASIL, AM, rodovia Manaus-Itacoatiara, Km232, pastagem 5 anos, A.G. Bandeira, 28.V.1977”: 33 soldiers; MPEG 142. "BRASIL, AM, rodovia Manaus-Itacoatiara, Km-232, capoeira, A.G.Bandeira, 22.V.1977”: 20 soldiers; MPEG 127. "BRASIL, AM, rodovia Manaus-Itacoatiara, Km-232, pastagem 1 ano, A.G.Bandeira, 21.V.1977”: 27 soldiers; MPEG 88. "BRASIL, AM, km 232, rodovia ManausItacoatiara, capoeira, A.G. Bandeira, 30.IV.1977”, 12 soldiers, 3 workers; MPEG 63. "BRASIL, AM, Manaus-Itacoatiara, Km-109, M. Dantas, 15.X.1976”: 4 soldiers, 2 workers;

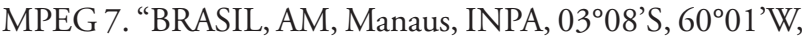
A.G.Bandeira, 18.VIII.1976”: 12 soldiers, 1 worker.

Genus Paraconvexitermes Cancello \& Noirot

- acangapua Cancello \& Noirot, 2003

Paratypes: MPEG 3449. "BRASIL, PA, Benevides, A. G. Bandeira, 29.VI.1981”: 5 soldiers, 2 workers; MPEG 3407. "BRASIL, PA, Belém, Mocambo, R. Constantino, 27.I.1989": 26 soldiers, 20 workers; MPEG 1446. "BRASIL, PA, Benevides, fazenda Morelândia, A. G. Bandeira, 02.VIII.1983": 9 soldiers, 9 workers; MPEG 1163. "BRASIL, PA, Benevides, fazenda Morelândia, A. G. Bandeira \& R. B. Neto, 27.VIII.1982": 16 soldiers, 7 workers; MPEG 1024. "BRASIL, PA, Benevides, fazenda Morelândia, Mata Primária, A. G. Bandeira \& R. B. Neto, 29.I.1981”: 5 soldiers, 15 
workers; MPEG 1010. "BRASIL, PA, Benevides, fazenda Morelândia, Mata Primária, A. G. Bandeira \& R. B. Neto, 27.I.1981": 10 soldiers, 4 workers; MPEG 982. "BRASIL, PA, Benevides, floresta, A. G. Bandeira, 16.IX.1980": 22 soldiers, 27 workers; MPEG 958. "BRASIL, PA, Benevides, Fazenda Morelândia, Capoeira, A. G. Bandeira, 18.VIII.1980": 16 soldiers, 16 workers; MPEG 793. "BRASIL, PA, Belém, Icoaraci, EIDAI, Paulo Emerita, 22.VI.1979”: 15 soldiers, 5 workers.

\section{Genus Subulitermes Holmgren}

- constricticeps Constantino, 1991a

Holotype: Soldier. MPEG 2846. "BRASIL, AM, Maraã, rio Japurá, R. Constantino, 12.X.1988”.

Paratypes: MPEG 2846. The same data as the holotype: 4 soldiers, 15 workers.

Genus Syntermes Holmgren

- barbatus Constantino, 1995

Holotype: Soldier. MPEG 1255. "BRASIL, Brasília, DF, fazenda Água Limpa, H. R. Coles, 30.IV.1977; S. brevimalatus, det.: H. R. Coles, sd.”.

Paratypes: MPEG 1255. The same data as the holotype: 8 soldiers, 1 worker; MPEG 1291. Idem: 19 soldiers.

- longiceps Constantino, 1995

Paratypes: MPEG 988. "BRASIL, PA, Benevides, fazenda Morelândia, floresta, R. B. Neto, 17.IX.1980”: 1 soldiers, 4 workers.

- nanus Constantino, 1995

Paratypes: MPEG 3895. "BRASIL, MT, Cuiabá, J. C. Dalponte, 11.IX.1982”: 12 soldiers, 73 workers; MPEG 3736. "BRASIL, AM, Humaitá, R. Constantino, 18.IX.1990": 13 soldiers, 10 workers; MPEG 2327. "BRASIL, MT, Cáceres, M. Zanuto, 02. III.1985": 15 soldiers, 6 workers. 1804).

- robustus Constantino, 1991 = spinosus (Latreille,

Holotype: Soldier. MPEG 2885. "BRASIL, AM, Maraã, rio Japurá, R. Constantino, 17.X.1988”.

Paratypes: MPEG 2885. The same data as the holotype: 20 soldiers, 60 workers, 17 imagoes (8 $\hat{\jmath}, 9$ ㅇ).

- tanygnathus Constantino, 1995

Holotype: Soldier. MPEG 3805. "BRASIL, AM, rio Tiquié, afluente rio Vaupés, Aldeia São João, próximo a PariCachoeira, R. B. Barthem, I.1991".

Paratypes: MPEG 3805. The same data as the holotype: 7 soldiers.

\section{SUBFAMILY TERMITINAE}

Genus Amitermes Silvestri

- aporema Constantino, 1992b

Holotype: Soldier. MPEG 3229. "BRASIL, AP, Aporema, R. Constantino, 26.X.1989".

Paratypes: MPEG 3229. The same data as the holotype: 18 soldiers, 103 workers; MPEG 3232. Idem: 7 soldiers, 89 workers.

Note: Lot number 3230 is listed as paratype (Constantino $1992 \mathrm{~b})$, but this sample was not found in the MPEG collection.

Genus Neocapritermes Holmgren

- pumillis Constantino, 1991c

Holotype: Soldier. MPEG 3360. "BRASIL, PA, Belém, APEG, R. Constantino, 30.VIII.1989”.

Paratypes: MPEG 3360. The same data as the holotype: 1 soldier, 14 workers; MPEG 3737. "BRASIL, AM, Humaitá, R. Constantino, 15.IX.1990": 2 soldiers, 1 worker.

- unicornis Constantino, 1991c

Holotype: Soldier. MPEG 3282. "BRASIL, AP, Serra do Navio, R. Constantino, 02 XI.1989”.

Paratypes: MPEG 3282. The same data as the holotype: 3 soldiers, 15 workers; MPEG 3270. Idem: 1 soldier, 8 workers; MPEG 3719. "BRASIL, AM, Humaitá, R. Constantino, 15.IX.1990": 1 soldier, 2 workers.

\section{Genus Orthognathotermes Holmgren}

- humilis Constantino, 1991a

Holotype: Soldier. MPEG 2825. "BRASIL, AM, Maraã, rio Japurá, R. Constantino, 11.X.1988”.

Paratypes: MPEG 2825. The same data as the holotype: 14 soldiers, 20 workers.

Genus Spinitermes Wasmann

- longiceps Constantino, 1991a

Holotype: Soldier. MPEG 2835. "BRASIL, AM, Maraã, rio Japurá, R. Constantino, 12.X.1988”.

Paratypes: MPEG 2835. The same data as the holotype: 14 soldiers, 28 workers, 50 imagoes ( 27 ふै, 23 † ). 
Table 1 - Geographical coordinates of the type-localities of Isoptera in the MPEG collection.

\begin{tabular}{|c|c|}
\hline Locality & Geographical Coordinates \\
\hline \multicolumn{2}{|l|}{ Amapá State } \\
\hline Aporema & $01^{\circ} 11^{\prime} \mathrm{N}, 52^{\circ} 49^{\prime} \mathrm{W}$ \\
\hline Macapá & $\begin{array}{l}00^{\circ} 02^{\prime} \mathrm{S}, 50^{\circ} 48^{\prime} \mathrm{W} \\
00^{\circ} 02^{\prime} \mathrm{S}, 52^{\circ} 32^{\prime} \mathrm{W}\end{array}$ \\
\hline Serra do Navio & $\begin{array}{l}00^{\circ} 59^{\prime} \mathrm{N}, 52^{\circ} 03^{\prime} \mathrm{W} \\
01^{\circ} 00^{\prime} \mathrm{N}, 52^{\circ} 04^{\prime} \mathrm{W}\end{array}$ \\
\hline \multicolumn{2}{|l|}{ Amazonas State } \\
\hline Fazenda Aruanã - Itacoatiara & $03^{\circ} 04^{\prime} \mathrm{S}, 58^{\circ} 45^{\prime} \mathrm{W}$ \\
\hline Maraã & $01^{\circ} 51^{\prime} \mathrm{S}, 65^{\circ} 27^{\prime} \mathrm{W}$ \\
\hline Aldeia São João (near Pari Cachoeira) & $00^{\circ} 10^{\prime} \mathrm{N}, 69^{\circ} 46^{\prime} \mathrm{W}$ \\
\hline \multicolumn{2}{|l|}{ Distrito Federal } \\
\hline Fazenda Água Limpa & $15^{\circ} 56^{\prime} \mathrm{S}, 47^{\circ} 54^{\prime} \mathrm{W}$ \\
\hline \multicolumn{2}{|l|}{ Pará State } \\
\hline Belém (APEG) & $01^{\circ} 27^{\prime} \mathrm{S}, 48^{\circ} 28^{\prime} \mathrm{W}$ \\
\hline Fazenda Morelândia - Benevides & $01^{\circ} 29^{\prime} \mathrm{S}, 48^{\circ} 13^{\prime} \mathrm{W}$ \\
\hline Serra Norte - Carajás & $06^{\circ} 00^{\prime} \mathrm{S}, 51^{\circ} 20^{\prime} \mathrm{W}$ \\
\hline
\end{tabular}

\section{ACKNOWLEDGMENTS}

Dr. Orlando Tobias Silveira, curator of the MPEG Invertebrate Collection, kindly facilitated access to the collection under his care. The authors thank Drs. Márcio Luís Leitão Barbosa, William Leslie Overal, Reginaldo Constantino, José Albertino Rafael, and Augusto Loureiro Henriques for critically reading the manuscript. The first author thanks the Brazilian "Conselho Nacional de Desenvolvimento Científico e Tecnológico" (CNPq) for financial support.

\section{LITERATURE CITED}

Bandeira, A.G.; Fontes, L.R. 1979. Nasutitermes acangussu, a new species of termites from Brazil (Isoptera, Termitidae, Nasutitermitinae). Revista Brasileira de Entomologia, 23: 119122.

Cancello, E.M. 1987. Observation on Cyranotermes Araujo, with a description of $C$. caete, new species (Isoptera, Termitidae, Nasutitermitinae). Papéis Avulsos de Zoologia, São Paulo, 36: 251-255.

Cancello, E.M.; Noirot, C. 2003. Paraconvexitermes acangapua (Isoptera: Termitidae: Nasutitermitinae), a new genus and new species of the so-called "small Neotropical soil-feeding nasutes" from South America. Ann. Soc. Entomolo. Fr., 39: 187-193.
Constantino, R. 1990a. Two new species of termites (Insecta, Isoptera) from western Brazilian Amazonia. Bol. Mus. Para. Emílio Goeldi, sér. Zool., 6: 3-10.

Constantino, R. 1990b. Agnathotermes crassinasus, new species of termite from the Amazon basin (Isoptera: Termitidae: Nasutitermitinae). Bol. Mus. Para. Emílio Goeldi, sér. Zool., 6: 43-46.

Constantino, R. 1990c. Anhangatermes macarthuri, new genus and species of soil-feeding nasute termite from Amapá State, Brazil (Isoptera: Termitidae: Nasutitermitinae). Goeldiana Zoologia, 3: $1-6$.

Constantino, R. 1990d. Notes on Cyranotermes Araujo with description of a new species (Isoptera, Termitidae, Nasutitermitinae). Goeldiana Zoologia, 2: 1-11.

Constantino, R. 1991a. Termites (Insecta, Isoptera) from the lower Japurá River, Amazonas State, Brazil. Bol. Mus. Para. Emílio Goeldi, sér. Zool., 7: 189-224.

Constantino, R. 1991b. Ereymatermes rotundiceps, new genus and species of termites from the Amazon Basin (Isoptera: Termitidae: Nasutitermitinae). Goeldiana Zoologia, 8: 1-11.

Constantino, R. 1991c. Notes on Neocapritermes Holmgren, with description of two new species from the Amazon Basin (Isoptera, Termitidae, Termitinae). Goeldiana Zoologia, 7: 1-13.

Constantino, R. 1992a. Notes on Embiratermes Fontes (Isoptera, Termitidae, Nasutitermitinae), with descriptions of two new species from Amapá State, Brazil. Bol. Mus. Para. Emílio Goeldi, sér. Zool., 8: 329-336.

Constantino, R. 1992b. A new species of Amitermes Silvestri from Amapá State, Brazil (Isoptera, Termitidae, Termitinae). Bol. Mus. Para. Emílio Goeldi, sér. Zool., 8: 337-341.

Constantino, R. 1995. Revision of the Neotropical genus Syntermes Holmgren (Isoptera: Termitidae). University of Kansas Science Bulletin, 55: 455-518.

Constantino, R.; Acioli, A.N.S.; Schmidt, K.; Cuezzo, C.; Carvalho, S.H.C. \& Vasconcellos, A. 2006. A taxonomic revision of the Neotropical termite genera Labiotermes Holmgren and Paracornitermes Emerson (Isoptera: Termitidae: Nasutitermitinae). Zootaxa, 1340:1 - 44.

Ride, W.D.L.; Cogger, H.G.; Dupuis, C.; Kraus, O.; Minelli, A.; Thompson, F.C.; Tubbs, P.K. (eds). 1999. International Code of Zoological Nomenclature. Fourth ed. International Trust for Zoological Nomenclature. The Natural History Museum, London, UK.

Recebido em 17/10/2005

Aceito em 25/07/2007 